\title{
Merging and alignment of Dirac points in a shaken honeycomb optical lattice
}

\author{
Selma Koghee, ${ }^{1,2}$ Lih-King Lim, ${ }^{2}$ M. O. Goerbig, ${ }^{2}$ and C. Morais Smith ${ }^{1}$ \\ ${ }^{1}$ Institute for Theoretical Physics, Utrecht University, Leuvenlaan 4, 3584 CE Utrecht, The Netherlands \\ ${ }^{2}$ Laboratoire de Physique des Solides, CNRS UMR 8502, Université Paris-Sud, 91405 Orsay, France
}

(Received 25 November 2011; published 27 February 2012)

\begin{abstract}
Inspired by the recent creation of a honeycomb optical lattice and the realization of a Mott-insulating state in a square lattice by shaking, we study here the shaken honeycomb optical lattice. For a periodic shaking of the lattice, Floquet theory may be applied to derive a time-independent Hamiltonian. In this effective description, the hopping parameters are renormalized by a Bessel function, which depends on the shaking direction, amplitude, and frequency. Consequently, the hopping parameters can vanish and even change sign, in an anisotropic manner, thus yielding different band structures. Here, we study the merging and the alignment of Dirac points and dimensional crossovers from the two-dimensional system to one-dimensional chains and zero-dimensional dimers. We also consider next-nearest-neighbor hopping, which breaks the particle-hole symmetry and leads to a metallic phase when it becomes dominant over the nearest-neighbor hopping. Furthermore, we include weak repulsive on-site interactions and find the density profiles for different values of the hopping parameters and interactions, both in a homogeneous system and in the presence of a trapping potential. Our results may be experimentally observed by use of momentum-resolved Raman spectroscopy.
\end{abstract}

DOI: 10.1103/PhysRevA.85.023637

PACS number(s): 67.85.-d, 73.22.Pr

\section{INTRODUCTION}

The study of Dirac points, i.e., the contact points between different energy bands with an approximately linear dispersion relation, has become a major issue since the experimental breakthrough in graphene-based electronics [1,2]. Indeed, the low-energy electronic properties of graphene are governed by a pseudorelativistic two-dimensional (2D) Dirac equation for massless fermions situated at the $K$ and $K^{\prime}$ corners of the Brillouin zone [3]. The Dirac points are topologically protected, and a gap is opened only when the inversion symmetry of the lattice or the time-reversal symmetry is broken.

The possibility of generating topological phase transitions in graphenelike systems has recently attracted a great deal of attention. Within a tight-binding description, an anisotropy in the nearest-neighbor hopping parameters makes the Dirac points move away from the high-symmetry $K$ and $K^{\prime}$ points and, under appropriate conditions, merge at time-reversalinvariant points in the first Brillouin zone [4-6]. Most saliently, this merging of Dirac points is associated with a topological phase transition between a semimetallic phase and a gapped band-insulating phase. An experimental investigation of the merging transition in graphene turns out to be problematic, since in order to appropriately modify the hopping parameters, an unphysically large strain needs to be applied to the graphene sheet [7].

An alternative system for the study of such topological transitions is that of ultracold atoms trapped in a honeycomb optical lattice. Since the seminal realization of the superfluidMott-insulator transition in the Bose-Hubbard model, ultracold atoms in optical lattices have become promising systems to emulate condensed-matter physics. Indeed, the lattice geometry, the dimensionality, and the atomic species, as well as the interactions, can be engineered with a high degree of precision. The more involved triangular and honeycomb geometries were recently realized experimentally, and exotic correlated states of matter have been observed experimentally [8] or predicted theoretically [9-12].

The application of a time-periodic perturbation on the optical lattice introduces yet another parameter scale into the system. A periodic shaking of the optical lattice, up to the kilohertz frequency range, has been implemented by placing one of the mirrors used to create the optical lattice on a piezoelectric material, such that the mirror can be moved back and forth in the direction of the beam $[13,14]$. The Floquet formalism shows that the hopping energy of the atoms in the shaken lattice is renormalized by a Bessel function, as a function of the shaking frequency and amplitude, thus allowing both the magnitude and the sign of the hopping parameter to change. This rather counterintuitive phenomenon, as compared to the standard tight-binding physics, has been experimentally observed in a one-dimensional cold-atomic system [14].

In this paper, we consider ultracold fermions trapped in a shaken honeycomb optical lattice. Within the Floquet formalism, we derive an effective Hamiltonian that generalizes that of a graphenelike material under strain. In particular, we find that the alignment and merging of Dirac points in momentum space are now accessible with ultracold fermions in the shaken optical lattice, and the phase diagram consists of various phases of the corresponding solid-state system that are otherwise difficult to realize. Furthermore, by taking into account a Hubbard-like interaction for spinful fermions, we study the density profiles for the homogeneous and the trapped gases within Hartree-Fock theory.

The outline of this paper is the following: In Sec. II A we introduce the time-dependent Hamiltonian and in Sec. II B we derive the time-independent one, by applying the Floquet formalism. In Sec. III we investigate the merging and alignment of Dirac points, when the optical lattice is shaken along specific directions. The description is extended to include interactions in Sec. IV, where we derive the dependence of the density on the chemical potential. Implications of our results for 
experiments are discussed in Sec. V. Finally, our conclusions are presented in Sec. VI.

\section{THE SHAKEN HONEYCOMB LATTICE}

In this section, we derive a time-independent effective description for ultracold atoms trapped in a periodically shaken honeycomb optical lattice by utilizing Floquet theory. For simplicity, we focus on a system of single-component fermionic atoms and consider only single-particle terms in this section. The results from the Floquet theory are valid for fermionic atoms with internal degrees of freedom as well as for bosonic atoms. In particular, the hyperfine state of fermionic atoms, playing the role of an effective spin-1/2 degree of freedom for electrons, will be considered when interaction effects are taken into account in Sec. IV.

\section{A. Time-dependent Hamiltonian}

In the tight-binding limit, the system of ultracold fermionic atoms trapped in a 2D shaken honeycomb optical lattice can be described by the Hamiltonian

$$
H(t)=H_{0}+W(t),
$$

which consists of two distinct parts. The static part

$$
\begin{aligned}
H_{0}= & -\gamma \sum_{j=1}^{3} \sum_{\mathbf{r} \in A}\left(a_{\mathbf{r}}^{\dagger} b_{\mathbf{r}+\mathbf{d}_{j}}+b_{\mathbf{r}+\mathbf{d}_{j}^{\dagger}}^{\dagger} a_{\mathbf{r}}\right) \\
& -\gamma^{\prime} \sum_{i=1}^{3} \sum_{j=1, j \neq i}^{3}\left(\sum_{\mathbf{r} \in A} a_{\mathbf{r}}^{\dagger} a_{\mathbf{r}+\mathbf{d}_{i}-\mathbf{d}_{j}}+\sum_{\mathbf{r} \in B} b_{\mathbf{r}}^{\dagger} b_{\left.\mathbf{r}+\mathbf{d}_{i}-\mathbf{d}_{j}\right)}\right. \\
& -\mu\left(\sum_{\mathbf{r} \in A} a_{\mathbf{r}}^{\dagger} a_{\mathbf{r}}+\sum_{\mathbf{r} \in B} b_{\mathbf{r}}^{\dagger} b_{\mathbf{r}}\right)
\end{aligned}
$$

is simply the tight-binding Hamiltonian in the honeycomb lattice, where $a_{\mathbf{r}}^{\dagger}\left(b_{\mathbf{r}}^{\dagger}\right)$ and $a_{\mathbf{r}}\left(b_{\mathbf{r}}\right)$ are, respectively, fermionic creation and annihilation operators on the lattice site $\mathbf{r}$ in the $A$ (B) sublattice. The three vectors

$$
\mathbf{d}_{1}=d \hat{e}_{x}, \mathbf{d}_{2}=\frac{d}{2}\left(-\hat{e}_{x}+\sqrt{3} \hat{e}_{y}\right), \mathbf{d}_{3}=\frac{d}{2}\left(-\hat{e}_{x}-\sqrt{3} \hat{e}_{y}\right),
$$

connect an $A$-lattice site with its three nearest-neighbor (NN) $B$-lattice sites and are given in terms of the distance $d=$ $8 \pi / 3 \sqrt{3} k$ between NN sites, where $k$ is the laser wave number (see Fig. 1). Here, $\gamma, \gamma^{\prime}>0$ characterize the energy gained in hopping to the NN and next-nearest-neighbor (NNN) sites, respectively, and $\mu$ is the on-site energy. We remark that the NNN hopping is taken into account because the NN hopping may be rendered vanishingly small in the effective timeindependent description. In this regime, the NNN hopping may become the dominant kinetic term. In a square lattice, where the potential is separable into independent $\hat{e}_{x}$ and $\hat{e}_{y}$ components, the NNN hopping is identically zero [15]. However, the NNN hopping can be nonzero in the honeycomb lattice, since its potential is not separable into $\hat{e}_{x}$ and $\hat{e}_{y}$ components. Nevertheless, it may be expressed as the sum of two triangular lattices.

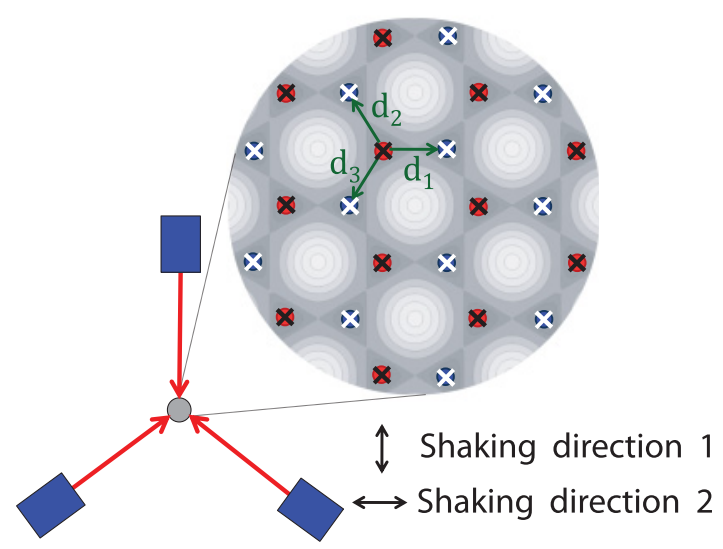

FIG. 1. (Color online) Laser configuration used to create the honeycomb lattice, which consists of two triangular sublattices $(A$, black crosses, and $B$, white crosses). The vectors $\mathbf{d}_{1}, \mathbf{d}_{2}$, and $\mathbf{d}_{3}$ connect a site on the $A$ sublattice to its nearest neighbors on the $B$ sublattice.

The time-dependent part of the Hamiltonian (1),

$$
W(t)=m \Omega^{2} \cos (\Omega t)\left(\sum_{\mathbf{r} \in A} \mathbf{r} \cdot \rho a_{\mathbf{r}}^{\dagger} a_{\mathbf{r}}+\sum_{\mathbf{r} \in B} \mathbf{r} \cdot \rho b_{\mathbf{r}}^{\dagger} b_{\mathbf{r}}\right),
$$

describes the harmonic shaking of the lattice in the direction $\rho$ with a driving frequency $\Omega$ in the comoving frame, as described in Ref. [16]. As a consequence of the transformation to the comoving frame, $W(t)$ describes atoms of mass $m$ experiencing a position-dependent sinusoidal force.

\section{B. Effective Hamiltonian}

The unavoidable complexity that arises when dealing with a quantum many-body system out of equilibrium has recently motivated the development of new theoretical tools, for example, the time-dependent density matrix renormalization group [17], time-dependent dynamical mean-field theory [18], and exact diagonalization [19]. However, for a periodically driven quantum system, the Floquet theory offers a simplified description of the system, in the form of a time-independent effective Hamiltonian, if the period $T=2 \pi / \Omega$ is the shortest time scale in the problem [20]. In this limit, the atoms cannot follow the shaking motion adiabatically and remain thus at their average lattice positions, albeit with renormalized hopping parameters. The system is thus considered to be in a stationary state and knowledge of the equilibrium physics can be employed.

Let us consider the Floquet Hamiltonian defined by $H_{F}=$ $H(t)-i \hbar \partial_{t}$, where $H(t+T)=H(t)$ is periodic in time [20]. The eigenvalue equation is then given by

$$
H_{F}|\phi(q, t)\rangle=\epsilon_{\phi}|\phi(q, t)\rangle,
$$

where $\epsilon_{\phi}$ is the quasienergy defined uniquely up to a multiple of $\hbar \Omega$. Any solution $|\phi(q, t)\rangle$ is part of a set of solutions $\exp (i n \Omega t)|\phi(q, t)\rangle$ with integer $n$, which all correspond to the same physical solution. Hence, the spectrum of the Floquet Hamiltonian possesses a Brillouin-zone-like structure [20]. The interest therefore lies with the states in the first Brillouin zone, i.e., states with quasienergies $-\hbar \Omega / 2<\epsilon_{\phi} \leqslant \hbar \Omega / 2$. 
The space in which the states $|\phi(q, t)\rangle$ are defined is a composite of the Hilbert space spanned by square integrable functions on configuration space, $|\alpha(q)\rangle$, and the space of $T$-periodic functions. The state $|\phi(q, t)\rangle$ may be written down in an orthonormal basis in the composite space according to

$$
|\phi(q, t)\rangle=\sum_{n=0}^{\infty} \sum_{\alpha} c_{n, \alpha} \exp [-i \hat{F}(t)+i n \Omega t]|\alpha(q)\rangle,
$$

where $c_{n, \alpha}$ are coefficients to normalize $|\phi(q, t)\rangle$ and the operator $\hat{F}(t)$ can be any $T$-periodic Hermitian operator. Therefore, we can conveniently choose $\hat{F}(t)$ to be $\hat{F}(t)=\hbar^{-1} \int_{0}^{t} d t^{\prime} W\left(t^{\prime}\right)$, such that $H(t)-\hbar \partial_{t} \hat{F}(t)=H_{0}$. If the condition

$$
\begin{aligned}
& \left\langle\alpha^{\prime}(q)\right|\left\langle\exp [i \hat{F}(t)] \exp \left[i\left(n-n^{\prime}\right) \Omega t\right] H_{0}\right. \\
& \quad \times \exp [-i \hat{F}(t)]\rangle_{T}|\alpha(q)\rangle \ll \hbar \Omega
\end{aligned}
$$

is satisfied for any two states $|\alpha(q)\rangle$ and $\left|\alpha^{\prime}(q)\right\rangle$, then the eigenvalues $\epsilon_{\phi}$ are approximately

$$
\begin{aligned}
\epsilon_{\phi} & =\left\langle\phi(q, t)\left|\left\langle H_{F}\right\rangle_{T}\right| \phi(q, t)\right\rangle \\
& \approx \sum_{\alpha, \alpha^{\prime}} c_{0, \alpha^{\prime}} c_{0, \alpha}\left\langle\alpha^{\prime}(q)\left|\left\langle\exp [i \hat{F}(t)] H_{0} \exp [-i \hat{F}(t)]\right\rangle_{T}\right| \alpha(q)\right\rangle .
\end{aligned}
$$

Here, $\langle\mathcal{O}(t)\rangle_{T}=T^{-1} \int_{0}^{T} d t \mathcal{O}(t)$ denotes the time average of the operator $\mathcal{O}(t)$ over the period $T$. The condition (7) will hold for $n \neq n^{\prime}$, if $H_{0}$ is nearly constant during the period $T$, which is small if $\Omega$ is large. In this case, states with different $n$ do not mix. If $\Omega$ is large enough, such that the condition (7) also holds for $n=n^{\prime}$, then the energy spectrum will split up into energy bands labeled by an index $n$, where the details within the energy band are determined by $H_{0}$. Because the states with different indices $n$ are separated by an energy which is a multiple of $\hbar \Omega$ and because the spectrum possesses a Brillouin-zone-like structure, only the terms with $n=0$ need to be taken into account. The effective Hamiltonian $H_{\text {eff }}$, which gives rise to the same spectrum as the Floquet Hamiltonian, is then defined by [21]

$$
\begin{aligned}
H_{\text {eff }} & =\left\langle\exp [i \hat{F}(t)] H_{0} \exp [-i \hat{F}(t)]\right\rangle_{T} \\
& =\left\langle\sum_{n=0}^{\infty} \frac{i^{n}}{n !}\left[\hat{F}(t), H_{0}\right]_{n}\right\rangle_{T} .
\end{aligned}
$$

Here, $[\hat{F}, \hat{G}]_{n}$ denotes the multiple commutator, which is defined by $[\hat{F}, \hat{G}]_{n+1}=\left[\hat{F},[\hat{F}, \hat{G}]_{n}\right]$ and $[\hat{F}, \hat{G}]_{0}=\hat{G}$.

Effective Hamiltonians corresponding to Eq. (9) have been derived for linear shaking of a one-dimensional lattice [22] and for elliptical shaking of a triangular lattice [23]. For the shaken honeycomb lattice studied here, the condition (7) is satisfied if $\gamma \ll \hbar \Omega$, and the effective Hamiltonian becomes

$$
\begin{aligned}
H_{\mathrm{eff}}= & -\sum_{j=1}^{3} \sum_{\mathbf{r} \in A} \gamma_{j}\left(a_{\mathbf{r}}^{\dagger} b_{\mathbf{r}+\mathbf{d}_{j}}+b_{\mathbf{r}+\mathbf{d}_{j}^{\dagger}}^{\dagger} a_{\mathbf{r}}\right)-\sum_{i=1}^{3} \sum_{j=1, j \neq i}^{3} \gamma_{i, j}^{\prime} \\
& \times\left(\sum_{\mathbf{r} \in A} a_{\mathbf{r}}^{\dagger} a_{\mathbf{r}+\mathbf{d}_{i}-\mathbf{d}_{j}}+\sum_{\mathbf{r} \in B} b_{\mathbf{r}}^{\dagger} b_{\left.\mathbf{r}+\mathbf{d}_{i}-\mathbf{d}_{j}\right)}\right. \\
& -\mu\left(\sum_{\mathbf{r} \in A} a_{\mathbf{r}}^{\dagger} a_{\mathbf{r}}+\sum_{\mathbf{r} \in B} b_{\mathbf{r}}^{\dagger} b_{\mathbf{r}}\right)
\end{aligned}
$$

where the renormalized $\mathrm{NN}$ hopping parameters $\gamma_{j}$ are given by

$$
\gamma_{j}=\gamma J_{0}\left(\left|\mathbf{d}_{j} \cdot \rho \frac{m \Omega}{\hbar}\right|\right),
$$

and the renormalized NNN hopping parameters by

$$
\gamma_{i, j}^{\prime}=\gamma^{\prime} J_{0}\left(\left|\left(\mathbf{d}_{i}-\mathbf{d}_{j}\right) \cdot \boldsymbol{\rho} \frac{m \Omega}{\hbar}\right|\right)
$$

(see the Appendix for detailed calculations). In these expressions, $J_{0}(x)$ denotes the zeroth-order Bessel function of the first kind, which shows a damped oscillation around zero.

In terms of the renormalized $\mathrm{NN}$ and $\mathrm{NNN}$ hopping parameters, the diagonalization of the effective Hamiltonian (10) yields the dispersion relation

$$
\epsilon_{\lambda}(\mathbf{q})=h(\mathbf{q})+\lambda|f(\mathbf{q})|,
$$

where $\lambda= \pm$ is the band index, and we have defined the functions

$$
f(\mathbf{q})=\sum_{j} \gamma_{j} \exp \left(-i \mathbf{q} \cdot \mathbf{d}_{j}\right)
$$

and

$$
h(\mathbf{q})=2 \sum_{i<j} \gamma_{i, j}^{\prime} \cos \left[\mathbf{q} \cdot\left(\mathbf{d}_{i}-\mathbf{d}_{j}\right)\right] .
$$

\section{MERGING AND ALIGNMENT OF DIRAC POINTS}

In this section, the honeycomb lattice with anisotropic hopping is studied. In the first two subsections, only NN hopping is considered for illustrative reasons. Indeed, this allows for a simple understanding of the main effects of shaking on Dirac-point motion and dimensional crossover. In Sec. IIIC, we discuss how the picture evolves when NNN hopping is included, and the sign of the hopping parameters is investigated in Sec. III D. Since the system with two equal NN hopping parameters and a single independent one captures the essential features of systems with three independent $\mathrm{NN}$ hopping parameters, we will focus on this system. The numbering of the $\gamma_{j}$ 's is chosen such that $\left|\gamma_{2}\right|=\left|\gamma_{3}\right|=\gamma_{2,3}$, which can be achieved by shaking in a direction parallel or perpendicular to $\mathbf{d}_{1}$.

Although the atoms in the optical lattice are charge-neutral objects, we shall adopt the language of condensed-matter physics and call a zero-gap phase with a pair of Dirac cones and a vanishing density of states at the band-contact points a semimetal, whereas a gapped phase is called a band insulator. Furthermore, NNN hopping induces a metallic phase for small values of $\gamma_{j}$ because of an overlap between the two bands that yields a nonvanishing density of states at the energy level of the band-contact points.

\section{A. Merging of Dirac points}

If the lattice is shaken in the direction perpendicular to $\mathbf{d}_{1}$ (direction 1 in Fig. 1), $\gamma_{1}$ remains equal to $\gamma$, whereas $\gamma_{2}$ and $\gamma_{3}$ are renormalized to a smaller value. An increase in the shaking amplitude results in a decrease in $\gamma_{2,3}=\gamma_{2}=\gamma_{3}$, which is depicted by the arrow $M$ in Fig. 2(a). When the 


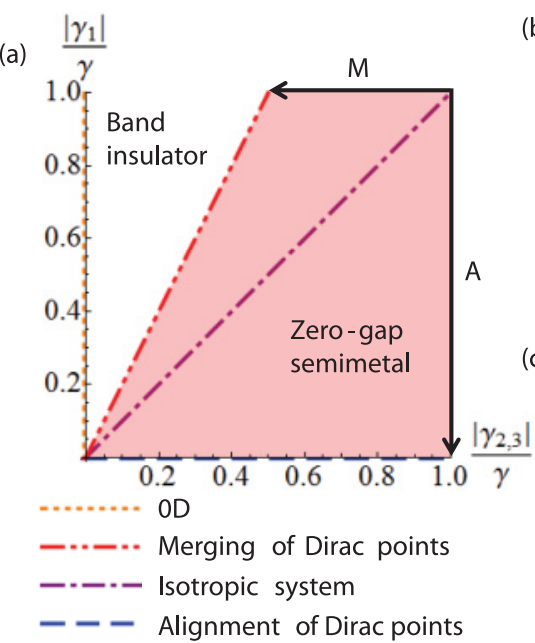

(b)

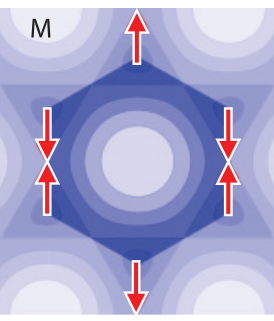

(c)

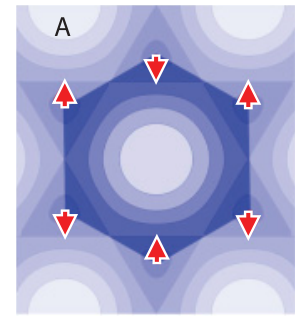

FIG. 2. (Color online) (a) Phase diagram, showing the phase transition between the zero-gap semimetallic phase and the insulating phase, which happens at $\gamma_{1}=2 \gamma_{2,3}$. Here, we have chosen $\gamma^{\prime}=0$. (b) Dirac-point motion in the first Brillouin zone for a shaking direction perpendicular to $\mathbf{d}_{1}$ [direction $M$ in the phase diagram (a)]. (c) Dirac-point motion in the first Brillouin zone for a shaking direction parallel to $\mathbf{d}_{1}$ [direction $A$ in the phase diagram (a)]. The contour plots depict the dispersion of the isotropic system with an arbitrary color scale. The area with higher contrast is the first Brillouin zone.

hopping parameters change according to this arrow $M$, the energy spectrum evolves from that of Fig. 3(a) to that shown in Fig. 3(b). The Dirac points, originally situated at the corners $K$ and $K^{\prime}$ of the first Brillouin zone, start to move in the $q_{y}$ direction along the vertical edges of the latter. This motion is depicted by the arrows in Fig. 2(b). Even though the two Dirac points are no longer located at the high-symmetry points $K$ and $K^{\prime}$, they remain related by time-reversal symmetry, such that their Berry phases $\pi$ and $-\pi$ are opposite. This nonzero Berry
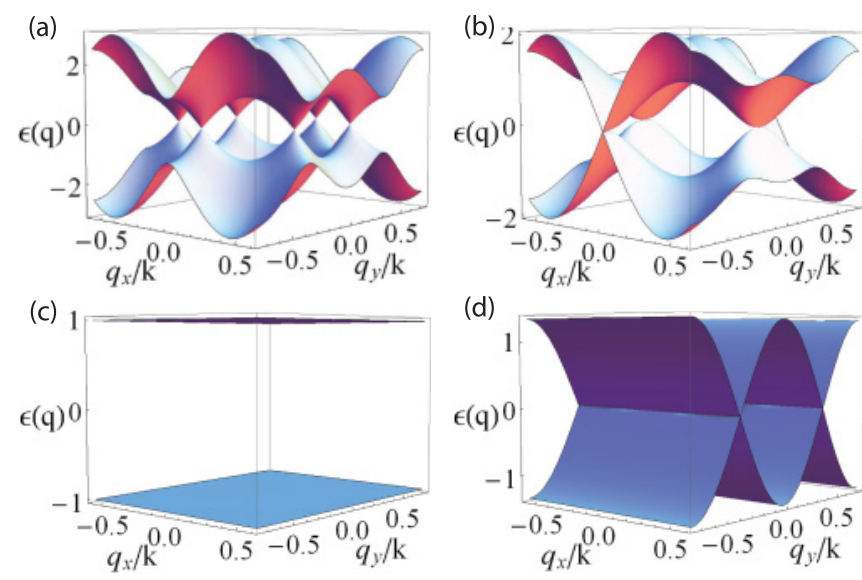

FIG. 3. (Color online) Energy dispersion (13) for the shaken honeycomb optical lattice in units of $\gamma$, with $\gamma^{\prime}=0$. The labels $q_{x}$ and $q_{y}$ represent the $x$ and $y$ components of the momentum, respectively. The $x$ and $y$ axes have been chosen such that the $\mathrm{NN}$ vectors are given by Eq. (3). (a) The isotropic case, where $\gamma_{1}=\gamma_{2,3}$. (b) The merged Dirac points, where $\gamma_{2,3}=\gamma_{1} / 2$. (c) The zero-dimensional case, where $\gamma_{2,3}=0$. (d) The aligned Dirac points, where $\gamma_{1}=0$. phase topologically protects each of the Dirac points and thus the semimetallic phase remains robust until $\gamma_{2,3}=\gamma_{1} / 2$, where the two points merge at a time-reversal-invariant momentum, i.e., half of a reciprocal lattice vector [6]. In the present example, this point is situated at the center of the vertical edges of the first Brillouin zone, and the band dispersion becomes parabolic in the $y$ direction while remaining linear in the $x$ direction [see Fig. 3(b)]. The merged Dirac points are no longer topologically protected due to the annihilation of the opposite Berry phases. Consequently, a further increase of the shaking amplitude, which results in a further decrease of $\gamma_{2,3}$, leads to the opening of a gap between the two bands. Thus, the system undergoes a topological phase transition from a semimetal to a band insulator. This merging transition was also studied in a static setup in Ref. [24], where it was proposed that the hopping amplitudes $\gamma$ were modified by a change in the intensity of one of the lasers used to create the optical lattice. In contrast to this static setup, shaking the honeycomb lattice allows one to completely annihilate some of the NN hopping parameters and even to change their sign. This sign change occurs at the zeros of the Bessel function [see Eq. (11)]. For an example system of ${ }^{40} \mathrm{~K}$ atoms in a lattice created by lasers with a wavelength of $830 \mathrm{~nm}$, which is shaken in the direction perpendicular to $\mathbf{d}_{1}$, the situation $\gamma_{2,3}=0$ is encountered for

$$
\rho=180 \mathrm{~nm}, \quad \Omega / 2 \pi=6 \mathrm{kHz},
$$

which corresponds to the first zero of the Bessel function. At this particular point, and if $\gamma^{\prime}=0$ in addition, the system consists of a set of effectively decoupled horizontal bonds along which the atoms are solely allowed to hop. This yields two flat bands at $\pm \gamma_{1}$ [see Fig. 3(c)] that may be viewed as the extreme limit of the band-insulating phase. Alternatively, one may view this situation upon decrease in the value of $\gamma_{2,3}$ as a dimensional crossover from a $2 \mathrm{D}$ band insulator to a zero-dimensional (0D) system. A small nonzero value of $\gamma_{2,3}$ simply provides a weak dispersion of these decoupled bands (not shown).

\section{B. Alignment of Dirac points}

Another dimensional crossover, from $2 \mathrm{D}$ to $1 \mathrm{D}$, may be obtained if the lattice is shaken in the direction parallel to one of the NN vectors (direction 2 in Fig. 1). Here, we choose $\mathbf{d}_{1}$ to maintain the symmetry $\gamma_{2,3}=\gamma_{2}=\gamma_{3}$. In this case, both $\gamma_{1}$ and $\gamma_{2,3}$ are renormalized by Bessel functions, albeit with different arguments. Since all hopping parameters are renormalized, the trajectory of the system in the phase space upon increase in the shaking amplitude is not a straight line, as was the case for shaking perpendicular to a hopping direction, and has a new feature: the alignment of Dirac points, which occurs for $\gamma_{1}=0$. The first zero of $\gamma_{1}$ is found at

$$
\rho=92 \mathrm{~nm}, \quad \Omega / 2 \pi=6 \mathrm{kHz}
$$

for the same system of ${ }^{40} \mathrm{~K}$ atoms mentioned above. Here, for illustrative purposes, a simplified trajectory of the system is depicted in Fig. 2(a) by arrow $A$, which corresponds to the motion of the Dirac points in reciprocal space as shown in Fig. 2(c). As $\gamma_{1}$ approaches zero, the Dirac points align in lines parallel to the $x$ axis at $q_{y}= \pm \pi / \sqrt{3} d$, and the energy barriers between the aligning points are lowered. Consequently, when 
$\gamma_{1}=0$, the energy spectrum contains lines where the two energy bands meet and the dispersion is linear, as is shown in Fig. 3(d). The dispersion relation (13) then reads simply

$$
\epsilon_{\lambda}(\mathbf{q})=2 \lambda \gamma_{2,3}\left|\cos \left(\frac{\sqrt{3}}{2} q_{y} d\right)\right|,
$$

and one clearly sees the 1D character. Indeed, there is no dispersion in the $q_{x}$ direction, as is also evident from Fig. 3(d), and the system may be viewed as completely decoupled 1D chains in which the zigzag arrangement is of no importance. In this particular limit, the sites $A$ and $B$ are therefore no longer inequivalent such that the unit cell is effectively divided by 2 , and the size of the first Brillouin zone is consequently doubled. The aligned Dirac points may thus, alternatively, be viewed as due to an artificial folding of the second (outer) half of the first Brillouin zone into its inner half. However, this aspect is very particular in that the Brillouin zone immediately retrieves its original size when $\gamma_{1}$ is small, but nonzero, or if NNN hoppings are taken into account. In both cases, one needs to distinguish the two different sublattices and one obtains a dispersion in the $q_{x}$ direction.

The actual behavior of the system for an increasing shaking amplitude is discussed in Sec. V. This behavior is more complicated because all three $\mathrm{NN}$ hopping parameters are renormalized, which, beyond the alignment, leads to the merging of Dirac points and the opening of a gap also in the case of shaking parallel to one of the NN vectors. In the absence of NNN hopping, the OD limit can be reached in addition.

\section{Next-nearest-neighbor hopping}

The major consequence of NNN hopping is to break particle-hole symmetry, as may be seen from Eq. (13), where nonzero values of $\gamma_{i, j}^{\prime}$ yield $\epsilon_{\lambda}(\mathbf{q}) \neq-\epsilon_{-\lambda}(\mathbf{q})$. Its relevance depends sensitively on the shaking direction, because of the different renormalization of the NN hopping parameters. The band structure with NNN hopping included is depicted in Fig. 4 for different shaking directions.

\section{Shaking in the direction perpendicular to $d_{1}$}

In the case of a shaking perpendicular to $\mathbf{d}_{1}$, only $\gamma_{2,3}$ are decreased, whereas $\gamma_{1}=\gamma$ remains the leading energy scale in the band structure. ${ }^{1}$ The band structure for the unshaken lattice is depicted in Fig. 4(a) for $\gamma^{\prime} / \gamma=0.1$, and one notices that the main features of the band structure, namely, the Dirac points, are unaltered with respect to the case $\gamma^{\prime}=0$ in Fig. 3(a), apart from the flattening of the upper band as compared to the lower one. When the merging transition $\gamma_{2,3}=\gamma_{1} / 2$ is approached, the value of which is determined by the zeros of $f(\mathbf{q})$ in Eq. (14) and therefore does not depend on the NNN hopping parameters, the bandwidth remains dominated by the largest hopping parameter $\gamma_{1}$, such that the band structure [Fig. 4(b)] at the transition is essentially the same

\footnotetext{
${ }^{1}$ We concentrate on $\mathbf{d}_{1}$ as a reference direction, but it may naturally be replaced by any other direction $\mathbf{d}_{j}$, in which case $\gamma_{j}=\gamma$ remains constant.
}
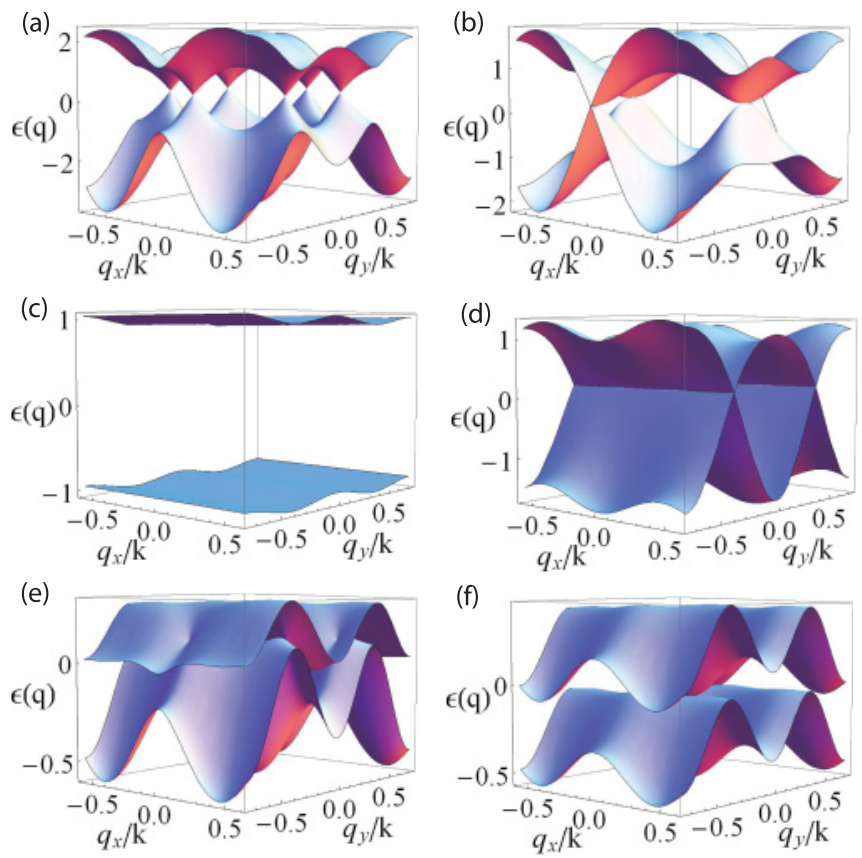

FIG. 4. (Color online) Energy dispersion (13) for the shaken honeycomb optical lattice in units of $\gamma$, with $\gamma^{\prime}=0.1 \gamma$. The labels $q_{x}$ and $q_{y}$ represent the $x$ and $y$ components of the momentum, respectively. The $x$ and $y$ axes have been chosen such that the NN vectors are given by Eq. (3). (a) The homogeneous case, where $\gamma_{1}=\gamma_{2,3}$. (b) The merged Dirac points, where $\gamma_{2,3}=\gamma_{1} / 2$. (c) The zero-dimensional case, where $\gamma_{2,3}=0$. (d) The aligned Dirac points, where $\gamma_{1}=0$. (e) An example of the metallic phase with $\rho=5.2(\hbar / m \Omega d) \hat{e}_{x}$. (f) Another example of the metallic phase with $\rho=4.8(\hbar / m \Omega d) \hat{e}_{x}$.

as in Fig. 3(b) for $\gamma^{\prime}=0$. In the OD limit, with $\gamma_{2,3}=0$ the originally flat bands [Fig. 3(c)] acquire the weak dispersion of a triangular lattice as a consequence of the nonzero NNN hopping parameters. However, as expected from the above arguments, the dispersion is on the order of $\gamma^{\prime}$ and thus small as compared to the energy separation $\sim 2 \gamma_{1}=2 \gamma$ between the two bands.

\section{Shaking in the direction parallel to $d_{1}$}

In contrast to a shaking direction perpendicular to $\mathbf{d}_{1}, \mathrm{NNN}$ hopping has more drastic consequences if the lattice is shaken in the direction parallel to $\mathbf{d}_{1}$. In this case, all $\mathrm{NN}$ hopping parameters are decreased, and the relative importance of NNN hopping is enhanced. Notice further that the NNN lattice vectors $\pm\left(\mathbf{d}_{2}-\mathbf{d}_{3}\right)$ are now perpendicular to the shaking direction such that $\gamma_{2,3}^{\prime}=\gamma^{\prime}=0.1 \gamma$ remains unrenormalized. Also in this case, the system is approaching the $1 \mathrm{D}$ limit, with $\gamma_{1}=0$ [see Fig. 4(d)]. However, in contrast to Fig. 3(d), here the chains remain coupled by NNN hopping which yields a dispersion in the $q_{x}$ direction. Furthermore, as mentioned above, the $A$ and $B$ sites are now not equivalent from a crystallographic point of view, such that the outer parts of the first Brillouin zone cannot be folded back into the inner one, as may be seen from Fig. 4(d).

Finally, for particular values of the shaking amplitude in the direction parallel to $\mathbf{d}_{1}$, the $\mathrm{NN}$ hopping parameters can be decreased in such a manner as to render $\gamma_{2,3}$ more relevant. In 
this case, the two bands can overlap in energy, as depicted in Figs. 4(e) and 4(f) for $\rho=5.2(\hbar / m \Omega d) \hat{e}_{x}$ (in which case $\gamma_{1} \approx$ $\gamma_{2,3}$ ) and $\rho=4.8(\hbar / m \Omega d) \hat{e}_{x}$ (with $\gamma_{2,3} \approx 0$ ), respectively. In the latter example there are no band-contact points, in spite of the overlap between the two bands, and the system would be in an insulating phase if NNN hopping terms were not taken into account. This overlap in energy between the two bands yields a nonzero density of states at any energy, such that the semimetallic (or insulating) phase vanishes and yields, at half filling, a metallic phase with particle and hole pockets in the first Brillouin zone.

\section{The signs of the hopping parameters}

As already alluded to in the previous sections, the shaking of a honeycomb lattice can lead to a sign change of the hopping parameters. Quite generally, Fig. 5 shows that changing the relative signs of the $\mathrm{NN}$ hopping parameters results in a translation of the energy spectrum in momentum space. This effect was also mentioned in Ref. [4]. Indeed, the relative signs determine at which of the four time-reversal-invariant momenta in the first Brillouin zone the merging of Dirac points and the semimetal-insulator transition take place when $\left|\gamma_{1}\right|=2\left|\gamma_{2,3}\right|$. However, the sign change of the NN hopping parameters can be transformed away by a gauge transformation [4]. Nevertheless, the sign of the NNN hopping parameter is important, since it determines whether the upper or the lower band is flattened.

\section{INTERACTIONS}

Until now, we have considered single-component fermionic atoms and, due to the Pauli principle, the absence of $s$-wave
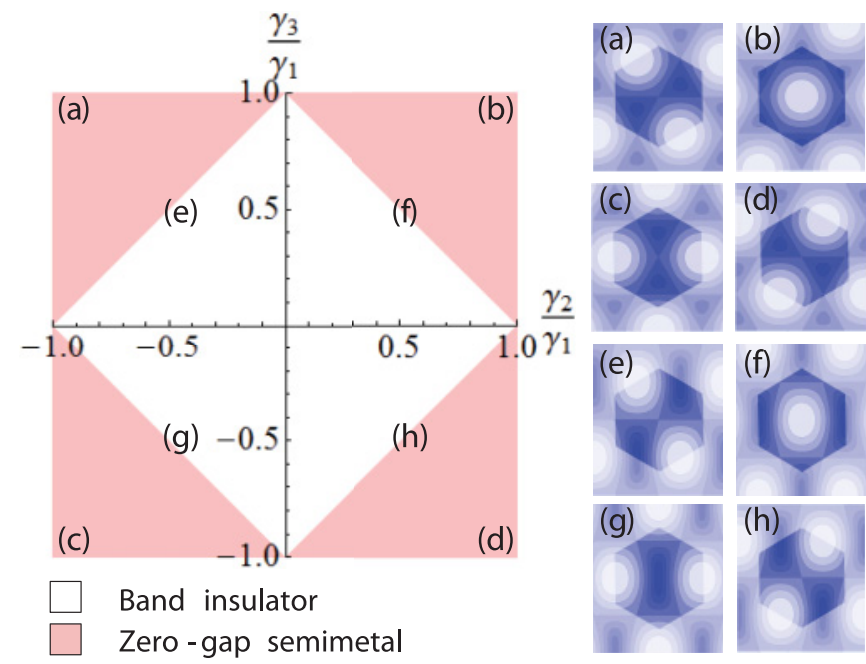

FIG. 5. (Color online) Phase diagram and contour plots of the energy bands, showing the effects of the renormalized NN hopping parameters $\gamma_{j}$. (a)-(h) Contour plots of the energy bands, where the color scaling is arbitrary and the first Brillouin zone is the area with higher contrast. The values of the NN hopping parameters for each contour plot are given by the positions of the corresponding letters in the phase diagram and $\gamma^{\prime}=0$. The dark regions indicate energies close to zero, whereas brighter regions are further away in energy from the Fermi level at half filling. interaction naturally results in an ideal Fermi lattice gas, albeit with an unusual band structure. By trapping two hyperfine states of the fermionic atoms, Hubbard-like interaction terms arise,

$$
\begin{aligned}
H_{\mathrm{int}}= & \sum_{\mathbf{r} \in A} \sum_{\sigma, \sigma^{\prime}} \frac{U}{2} a_{\mathbf{r}, \sigma}^{\dagger} a_{\mathbf{r}, \sigma^{\prime}}^{\dagger} a_{\mathbf{r}, \sigma^{\prime}} a_{\mathbf{r}, \sigma} \\
& +\sum_{\mathbf{r} \in B} \sum_{\sigma, \sigma^{\prime}} \frac{U}{2} b_{\mathbf{r}, \sigma}^{\dagger} b_{\mathbf{r}, \sigma^{\prime}}^{\dagger} b_{\mathbf{r}, \sigma^{\prime}} b_{\mathbf{r}, \sigma},
\end{aligned}
$$

where the fermionic operators now acquire an additional spin index $\sigma=\{\uparrow, \downarrow\}$, which is summed over, and $U$ is the interaction energy. Naturally, in order to be able to apply the Floquet theory in the presence of interactions, the Hamiltonian $H_{0}$ in Eq. (7) must now be replaced by $H=H_{0}+H_{\text {int }}$. This is the case in our study because we investigate the weak-coupling limit with $U \ll \gamma$. Since the interaction term commutes with the shaking, it is not renormalized, similarly to the on-site energy term proportional to $\mu$ in Eq. (2). However, it has been shown that complications may arise when a multiple of the energy $U$ is in resonance with a harmonic of $\hbar \Omega, m \hbar \Omega=n U$, for integer $m$ and $n$. Whereas the limit [25] $m \ll n$ is not considered here because it is in contradiction with the small- $U$ large-frequency limit, critical resonances may occur for $m \gg n$ [26]. Nevertheless, it has been shown in Ref. [26] that these resonances, which occur in higher-order perturbation theory, are strongly suppressed in the large- $m$ limit.

In the weakly interacting regime considered here, the ground state is adiabatically connected to that of the noninteracting system, with no broken symmetry. First, we use the Fourier transform of the creation and annihilation operators, $a_{\mathbf{r}, \sigma}=\mathcal{N}^{-1 / 2} \sum_{\mathbf{q}} \exp (i \mathbf{q} \cdot \mathbf{r}) a_{\mathbf{q}, \sigma}$, to find the Hamiltonian in momentum space. Within Hartree-Fock theory, we introduce a mean-field decoupling of the interaction terms,

$$
\begin{aligned}
a_{\mathbf{q} 1, \sigma}^{\dagger} & a_{\mathbf{q} 2, \sigma^{\prime}}^{\dagger} a_{\mathbf{q} 3, \sigma^{\prime}} a_{\mathbf{q} 4, \sigma} \\
\approx & \left\langle a_{\mathbf{q} 2, \sigma^{\prime}}^{\dagger} a_{\mathbf{q} 3, \sigma^{\prime}}\right\rangle a_{\mathbf{q} 1, \sigma}^{\dagger} a_{\mathbf{q} 4, \sigma}-\left\langle a_{\mathbf{q} 2, \sigma^{\prime}}^{\dagger} a_{\mathbf{q} 4, \sigma}\right\rangle a_{\mathbf{q} 1, \sigma}^{\dagger} a_{\mathbf{q} 3, \sigma^{\prime}} \\
& +a_{\mathbf{q} 2, \sigma^{\prime}}^{\dagger} a_{\mathbf{q} 3, \sigma^{\prime}}\left\langle a_{\mathbf{q} 1, \sigma}^{\dagger} a_{\mathbf{q} 4, \sigma}\right\rangle-a_{\mathbf{q} 2, \sigma^{\prime}}^{\dagger} a_{\mathbf{q} 4, \sigma}\left\langle a_{\mathbf{q} 1, \sigma}^{\dagger} a_{\mathbf{q} 3, \sigma^{\prime}}\right\rangle \\
& -\left\langle a_{\mathbf{q} 2, \sigma^{\prime}}^{\dagger} a_{\mathbf{q} 3, \sigma^{\prime}}\right\rangle\left\langle a_{\mathbf{q} 1, \sigma}^{\dagger} a_{\mathbf{q} 4, \sigma}\right\rangle+\left\langle a_{\mathbf{q} 2, \sigma^{\prime}}^{\dagger} a_{\mathbf{q} 4, \sigma}\right\rangle\left\langle a_{\mathbf{q} 1, \sigma}^{\dagger} a_{\mathbf{q} 3, \sigma^{\prime}}\right\rangle,
\end{aligned}
$$

such that the expectation values of both sides are equal. For the mean value we take

$$
\left\langle a_{\mathbf{q}, \sigma}^{\dagger} a_{\mathbf{q}^{\prime}, \sigma^{\prime}}\right\rangle=\left\langle b_{\mathbf{q}, \sigma}^{\dagger} b_{\mathbf{q}^{\prime}, \sigma^{\prime}}\right\rangle=\mathcal{N} n_{\mathbf{q}, \sigma} \delta_{\mathbf{q}, \mathbf{q}^{\prime}} \delta_{\sigma, \sigma^{\prime}},
$$

where $\mathcal{N}$ is the number of sites per sublattice, $n_{\mathbf{q}, \sigma}$ is the density of atoms with momentum $\mathbf{q}$ and spin index $\sigma$, and $\delta_{\alpha, \alpha^{\prime}}$ is the Kronecker delta. We then obtain the mean-field Hamiltonian

$$
H_{\mathrm{MF}}=H_{\mathrm{eff}}-\frac{U \mathcal{N} n^{2}}{8}+\frac{U n}{4} \sum_{\sigma, \mathbf{q}}\left(a_{\mathbf{q}, \sigma}^{\dagger} a_{\mathbf{q}, \sigma}+b_{\mathbf{q}, \sigma}^{\dagger} b_{\mathbf{q}, \sigma}\right),
$$

where the total density is defined by $n=\sum_{q, \sigma} n_{\mathbf{q}, \sigma}$. 
The Hamiltonian (22) may be rewritten in a matrix form:

$$
\begin{aligned}
H_{\mathrm{MF}}= & -\frac{U \mathcal{N} n^{2}}{8}+\sum_{\sigma, \mathbf{q}}\left(a_{\mathbf{q}, \sigma}^{\dagger} b_{\mathbf{q}, \sigma}^{\dagger}\right) \\
& \times\left(\begin{array}{cc}
h(\mu, \mathbf{q}) & f(\mathbf{q}) \\
f^{*}(\mathbf{q}) & h(\mu, \mathbf{q})
\end{array}\right)\left(\begin{array}{l}
a_{\mathbf{q}, \sigma} \\
b_{\mathbf{q}, \sigma}
\end{array}\right),
\end{aligned}
$$

where we have introduced the functions

$$
h(\mu, \mathbf{q})=\frac{U n}{4}-\mu-\gamma^{\prime} \sum_{i=1}^{3} \sum_{j=1, j \neq i}^{3} \exp \left[-i \mathbf{q} \cdot\left(\mathbf{d}_{i}-\mathbf{d}_{j}\right)\right],
$$

and $f(\mathbf{q})$ is defined in Eq. (14) The Hamiltonian (23) can then be diagonalized by the unitary operator

$$
\hat{\mathcal{U}}=\frac{1}{\sqrt{2}}\left(\begin{array}{cc}
1 & i f(\mathbf{q}) /|f(\mathbf{q})| \\
f^{*}(\mathbf{q}) /|f(\mathbf{q})| & -i
\end{array}\right),
$$

which yields

$$
\begin{aligned}
H_{\mathrm{MF}}= & -\frac{U \mathcal{N} n^{2}}{8}+\sum_{\sigma, \mathbf{q}}\left(c_{\mathbf{q}, \sigma}^{\dagger} d_{\mathbf{q}, \sigma}^{\dagger}\right) \\
& \times\left(\begin{array}{cc}
h(\mu, \mathbf{q})-|f(\mathbf{q})| & 0 \\
0 & h(\mu, \mathbf{q})+|f(\mathbf{q})|
\end{array}\right)\left(\begin{array}{c}
c_{\mathbf{q}, \sigma} \\
d_{\mathbf{q}, \sigma}
\end{array}\right) .
\end{aligned}
$$

Because the $c$ and $d$ quasiparticles are free, the partition function corresponding to the Hamiltonian (26) reads

$$
\begin{aligned}
Z= & \exp \left[\sum_{\sigma, \mathbf{q}}(\ln \{1+\exp [-\beta(h(\mu, \mathbf{q})-|f(\mathbf{q})|)]\}\right. \\
& +\ln \{1+\exp [-\beta(h(\mu, \mathbf{q})+|f(\mathbf{q})|)]\})],
\end{aligned}
$$

where $\beta=\left(k_{B} T\right)^{-1}$ with $k_{B}$ denoting the Boltzmann constant and $T$ the temperature.

The total number of particles $N$ is given by $(1 / \beta) \partial \ln Z / \partial \mu$, and one obtains

$$
\begin{aligned}
N= & \sum_{\sigma, \mathbf{q}}\left(\frac{1}{1+\exp [\beta(h(\mu, \mathbf{q})-|f(\mathbf{q})|)]}\right. \\
& \left.+\frac{1}{1+\exp [\beta(h(\mu, \mathbf{q})+|f(\mathbf{q})|)]}\right) .
\end{aligned}
$$

Since the expression inside the sum does not depend on spin, summing over $\sigma$ yields a factor of 2. One recognizes in Eq. (28) the Fermi-Dirac distribution function $N_{\mathrm{FD}}(x)=$ $[1+\exp (x)]^{-1}$. The number of particles $N$ is related to the density $n$, which is defined here as the number of particles per lattice site, i.e., $n=N / 2 \mathcal{N}$. Converting the sum over $\mathbf{q}$ into an integral, we derive the following self-consistent equation for the density:

$$
\begin{aligned}
n(\mu)= & \frac{1}{V_{1 \mathrm{BZ}}} \int_{1 \mathrm{BZ}} d^{2} \mathbf{q}\left\{N_{\mathrm{FD}}[\beta(h(\mu, \mathbf{q})-|f(\mathbf{q})|)]\right. \\
& \left.+N_{\mathrm{FD}}[\beta(h(\mu, \mathbf{q})+|f(\mathbf{q})|)]\right\},
\end{aligned}
$$

where the integral is restricted to the first Brillouin zone (1BZ), the surface of which is $V_{1 \mathrm{BZ}}$.
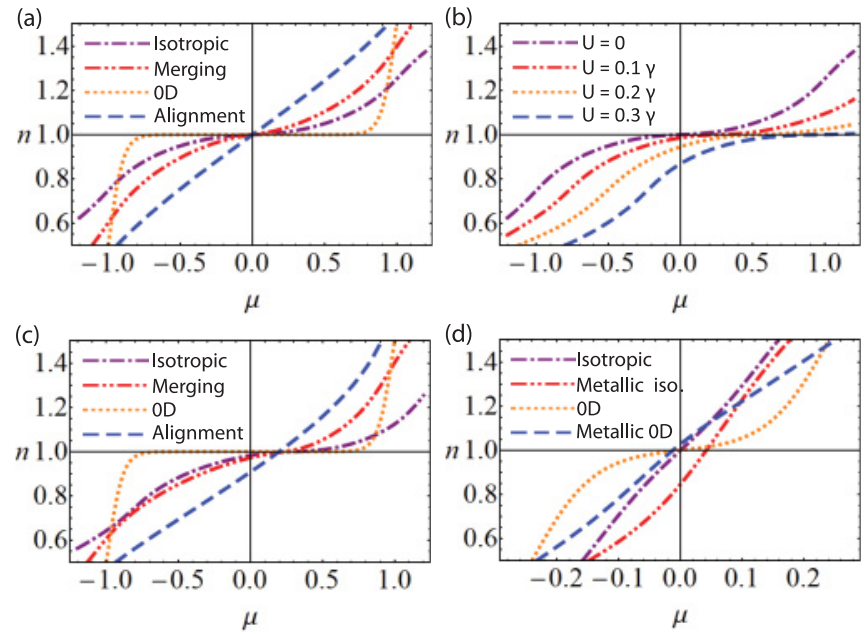

FIG. 6. (Color online) Density $n$ as a function of the chemical potential $\mu$. Unless specified otherwise in the figure, the NN hopping parameters $\gamma_{2,3}=\gamma_{1}=\gamma=1$, the NNN hopping parameter $\gamma^{\prime}=0$, the interaction strength $U=0$, and the inverse temperature $\beta=20$.

(a) Effect of the renormalization of the $\mathrm{NN}$ hopping parameters.

(b) Effect of the interaction strength $U$ for the isotropic case.

(c) Effect of the NNN hopping parameter $\gamma^{\prime}=0.1$ in the shaken lattice. (d) The metallic phase. For the isotropic cases, $\rho=$ $5.2(\hbar / m \Omega d) \hat{e}_{x}$, whereas for the 0D cases $\rho=4.8(\hbar / m \Omega d) \hat{e}_{x}$. These systems are in the metallic phase for $\gamma^{\prime}=0.1$, whereas for $\gamma^{\prime}=0$ they are in the semimetallic and the insulating phases, respectively.

In Fig. 6(a), the density $n(\mu)$ is plotted for several values of $\gamma_{2,3} / \gamma_{1}$. For the isotropic case, $\gamma_{2,3} / \gamma_{1}=1$, the result of $\mathrm{Zhu}$ et al. is reproduced [27]. For the 0D limit, the flat line due to the gap in the spectrum is clearly visible at the chosen temperature. Figure 6(b) confirms that repulsive interactions lead to a lower density than in a system without interactions for the same chemical potential. Figure 6(c) agrees with the observation that the NNN hopping breaks the particle-hole symmetry. This effect is also visible in Fig. 6(d), where the dependence of the density on the chemical potential is calculated for a shaking vector where the system is in the zero-gapped semimetallic phase for $\gamma^{\prime}=0$ and in the metallic phase for $\gamma^{\prime}=0.1$.

\section{POSSIBILITIES FOR EXPERIMENTAL OBSERVATION}

Honeycomb optical lattices have recently been realized experimentally, although the existing setups have only been used to investigate bosonic atoms [8,28]. Shaking of a lattice has been experimentally implemented in a one-dimensional lattice by a periodic modulation on the position of the reflecting mirrors [14]. For a honeycomb lattice, the shaking could be realized by means of an acousto-optical device, as proposed for a triangular lattice in Ref. [23].

The magnitude of the NN hopping parameter $\gamma$ in a honeycomb optical lattice has been evaluated in Ref. [24],

$$
\gamma \approx 1.861 E_{R}\left(\frac{V_{0}}{E_{R}}\right)^{3 / 4} \exp \left(-1.582 \sqrt{\frac{V_{0}}{E_{R}}}\right),
$$

in terms of the recoil energy $E_{R}=\hbar^{2} k^{2} / 2 m$ and the magnitude of the potential barrier between nearest-neighbor lattice sites, $V_{0}$. The magnitude of the NNN hopping parameter $\gamma^{\prime}$ is not 
(a) Perpendicular

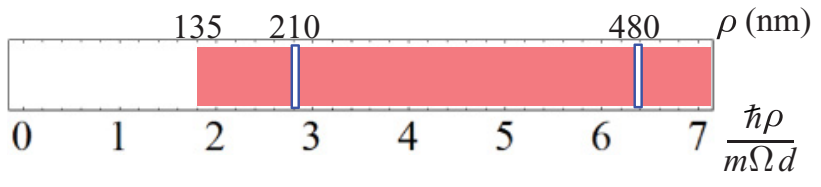

\section{\begin{tabular}{l|l|l} 
Gapped & Metallic $\quad$ OD
\end{tabular}}

(b) Parallel

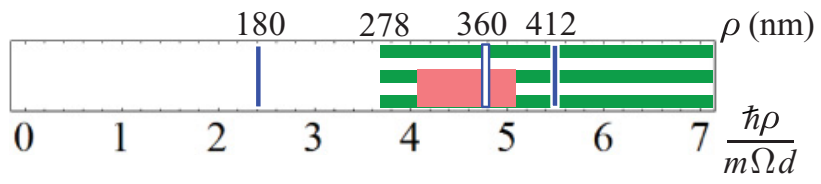

FIG. 7. (Color online) Overview of the different phases as a function of the shaking amplitude, starting from the semimetallic phase (blank part). The bottom scale gives the size of the argument of the Bessel function; the values for $\rho$ in nanometers correspond to the optical lattice discussed in Sec. III C with $\gamma^{\prime}=0.1 \gamma, \Omega / 2 \pi=6 \mathrm{kHz}$, a laser field with a wavelength of $830 \mathrm{~nm}$, and containing ${ }^{40} \mathrm{~K}$ atoms. Shaking (a) perpendicular and (b) parallel to one of the NN hopping directions.

yet known, but could be determined from numerical bandstructure calculations. In a typical experimental situation, we expect the ratio $\gamma^{\prime} / \gamma$ to be in the 5\%-10\% range, in agreement with the parameters chosen in the discussion of Sec. III C.

In a typical experiment, the shaking amplitude would be increased from zero to a finite value. Figure 7 shows the order in which the system goes through the different phases and dimensions as the shaking amplitude is increased. Here, the values of the shaking amplitude required for the dimensional crossovers are also given for the same system as discussed in Sec. III C and $\gamma^{\prime}=0.1 \gamma$. If the shaking direction is perpendicular to one of the $\mathrm{NN}$ vectors, the system will be in the gapped insulating phase beyond a certain value of the shaking amplitude, since the Bessel function crosses the value 0.5 only once and never obtains the value -0.5 . If the shaking is parallel to one of the $\mathrm{NN}$ vectors, the system will be in the metallic phase beyond a certain value of the shaking amplitude. Nevertheless, it is still possible to induce a merging of Dirac points and to open up a gap in the spectrum, since the NN hopping parameters are renormalized such that the value of one of them will in general differ from that of the other two. However, whether the system is actually driven into an insulating phase or remains metallic depends on the precise value of the ratio $\gamma^{\prime} / \gamma$.

In experiments, an overall harmonic trapping potential is imposed to confine the atoms. It is described by

$$
V_{\text {trap }}(\mathbf{r})=\frac{1}{2} m \omega_{\text {trap }}^{2} \mathbf{r}^{2},
$$

where $\omega_{\text {trap }}$ is the trapping frequency, and $\mathbf{r}$ is the position measured from the center of the trap. By applying the local density approximation, one finds that the chemical potential evolves radially according to $\mu \rightarrow \mu-V_{\text {trap }}\left(\mathbf{r}^{2}\right)$.

Figure 8(a) shows the density profile for several ratios of $\gamma_{2,3} / \gamma_{1}$, without NNN hopping or interactions. The case with
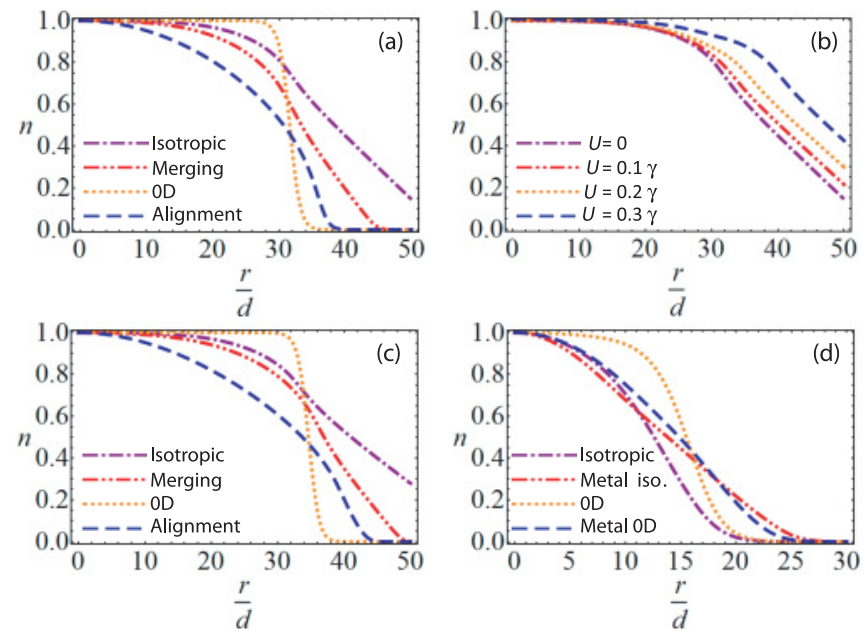

FIG. 8. (Color online) Density $n$ as a function of the distance from the trap's center, $r=|\mathbf{r}|$, which is expressed in units of the nearest-neighbor distance $d$. The trapping frequency has been chosen such that the trapping potential is given by $V_{\text {trap }}(\mathbf{r})=0.001 \gamma \mathbf{r}^{2} / d^{2}$. The chemical potential $\mu$ for each case has been chosen such that the density at the trap's center, $n$, is one particle per site. This corresponds to half filling, since we consider two species of fermions. Unless specified otherwise in the figure, the $\mathrm{NN}$ hopping parameters $\gamma_{2,3}=\gamma_{1}=\gamma=1$, the NNN hopping parameter $\gamma^{\prime}=0$, the interaction strength $U=0$, and the inverse temperature $\beta=20$.

(a) Effect of the renormalization of the $\mathrm{NN}$ hopping parameters.

(b) Effect of the interaction strength $U$ for the isotropic case. (c) Effect of the NNN hopping parameter $\gamma^{\prime}=0.1$ in the shaken lattice. (d) The metallic phase. For the isotropic cases, $\rho=$ $5.2(\hbar / m \Omega d) \hat{e}_{x}$, whereas for the 0D cases $\rho=4.8(\hbar / m \Omega d) \hat{e}_{x}$. These systems are in the metallic phase for $\gamma^{\prime}=0.1$, whereas for $\gamma^{\prime}=0$ they are in the semimetallic and the insulating phases, respectively.

$\gamma_{2,3} / \gamma_{1}=0$, when the system is in the extreme limit of the band-insulating phase, can be well distinguished from the other cases. Figure 8(b) shows that stronger interactions lead to a higher density away from the center of the trap. This effect becomes visible when the density starts to deviate from one particle per lattice site. Next-nearest-neighbor hopping leads to a higher density at the edge of the cloud compared to the case without NNN hopping, which can be seen by comparison of Figs. 8(a) and 8(c) and from Fig. 8(d). The latter shows the effect of NNN hopping on the density profile for the case where the NNN hopping gives rise to the metallic phase for two different shaking vectors. In the first case, $\rho=5.2(\hbar / m \Omega d) \hat{e}_{x}$, which gives $\gamma_{1} \approx \gamma_{2,3}$, such that without NNN hopping the system is in the zero-gapped semimetallic phase and the Dirac points are located very close to the corners of the first Brillouin zone. In the second case, $\rho=4.8(\hbar / m \Omega d) \hat{e}_{x}$, which results in $\gamma_{2,3} \approx 0$, such that without NNN hopping the system is in the insulating phase and the two energy bands are almost flat.

We emphasize that, in the present paper, we discuss only weak correlations that adiabatically affect the density. However, when the on-site interaction is increased further, one might expect correlated phases with inhomogeneous density, even at half filling. A detailed study of these correlated phases is a vast research issue that is yet ongoing and that is beyond the scope of the present paper. Here, we provide only a glimpse as to how the density, which we discussed 
above in the weak-coupling limit, may evolve in view of some phases studied in the literature. Mean-field calculations indicate a transition to an antiferromagnetic state above a value of $U / \gamma \simeq 2.2$ [9], whereas more sophisticated quantum Monte Carlo calculations indicate an intermediate spin-liquid phase between the semimetal and the antiferromagnetic phases [10]. The spin-liquid phase may be viewed as a Mott insulator with a charge localization on the lattice sites, and recent slave-rotor calculations indicate that such spin-liquid phases dominate the phase diagram for $\gamma_{1}>\gamma_{2,3}$ [11], which is the parameter range where the Dirac points would merge in the absence of interactions. The precise transition between the weakly interacting liquid phases and these strongly correlated Mott insulators could in principle be determined with the help of the above-mentioned density measurements.

A more promising technique for detecting Dirac-point motion is momentum-resolved Raman spectroscopy. This technique has been proposed as an equivalent of angleresolved photoemission spectroscopy for cold-atom systems [29]. It has not yet been realized experimentally, although momentum-resolved radio-frequency spectroscopy, which is a very similar technique, has already been implemented [30]. Notice further that another very similar technique, momentumresolved Bragg spectroscopy, has been applied to ultracold bosonic atoms in a static optical lattice by Ernst et al. [31]. Momentum-resolved spectroscopy can allow us to indirectly visualize the band structure. In momentum-resolved Raman spectroscopy, the system is irradiated with two laser pulses with frequencies $\omega_{1}$ and $\omega_{2}$. If the frequency difference is in resonance with a transition $\omega_{\mathrm{hf}}$ between atomic hyperfine states, $\omega_{1}-\omega_{2}=\omega_{\mathrm{hf}}$, some atoms are excited in a secondorder process to the higher hyperfine state. Then, with stateselective time-of-flight measurements, the dispersion of the atoms in the new state is measured, from which the dispersion of the original atoms can be derived. When the atoms are confined in a trapping potential and the laser pulses are focused on the center of the trap, the quality of the results obtained by Raman spectroscopy is comparable to those of a homogeneous system [29]. Furthermore, Raman spectroscopy yields better results for a system with strong interactions compared to standard time-of-flight measurements [29].

Notice that momentum-resolved Raman spectroscopy was originally proposed to be applied to a gas of ultracold fermionic atoms at equilibrium and not for a shaken lattice. We therefore discuss, in this final paragraph, why we think that this technique may also be applied to the present case. Naturally, as long as the frequencies of the additional lasers in the Raman-spectroscopy setup are small with respect to the shaking frequency, $\omega_{1}, \omega_{2} \ll \Omega$, even the full system satisfies the condition (7) for the validity of Floquet theory. As in the case of interactions, one needs, however, to avoid resonances between the different laser frequencies that could become critical [26]. The opposite limit, in which the laser frequencies and that of the hyperfine transition are larger than the shaking frequency, is more delicate. However, even then, the shaken system remains at quasiequilibrium as long as the intensities of the lasers used in Raman spectroscopy are weak, such that they constitute only a small perturbation. The atomic dynamics probed even at high frequencies is therefore still that of the atoms at quasiequilibrium, with the band strucure obtained from Floquet theory. Furthermore, in the experimental studies by Zenesini et al. [14], time-of-flight measurements were used to determine the momentum distribution of bosonic atoms in a shaken lattice. Apart from the time-scale considerations, there are also some length scales that need to be taken into account. There are indeed two requirements for the correct size of the focus of the laser beams. On the one hand, it needs to be larger than the lattice spacing, such that sufficiently many atoms can be excited, while on the other hand the focus of the beams should be small enough to have an approximately flat trapping potential inside the focus area. In addition, the choice of the length of the pulses could possibly be a problem, since for shorter pulses the excited atoms will be less affected by the lattice potential, whereas for longer pulses more atoms can be excited, leading to a stronger signal.

\section{CONCLUSIONS}

In conclusion, we have investigated the band engineering of fermionic atoms in an optical honeycomb lattice with the help of a periodic shaking of the lattice. If the shaking frequency $\Omega$ is large enough, i.e., if $\hbar \Omega$ constitutes the largest energy scale in the system, the Floquet theory may be applied and the system is at quasiequilibrium in the sense that the atoms cannot follow the rapid motion associated with the shaking. Depending on the direction of the shaking, one may render the hopping amplitudes in the quasistatic lattice anisotropic, due to a renormalization of the NN and NNN hopping parameters by Bessel functions that go through zero and change sign. As a consequence, dimensional crossovers can be induced in the absence of the NNN hopping. For a shaking direction parallel to one of the NN vectors (such as, e.g., $\mathbf{d}_{1}$ ), one can make one of the NN hopping parameters vanish, $\gamma_{1} \rightarrow 0$. The system then undergoes a transition from $2 \mathrm{D}$ to $1 \mathrm{D}$, while the Dirac points align simultaneously. Shaking in the perpendicular direction $\left(\perp \mathbf{d}_{1}\right)$ allows one to decrease two NN hopping amplitudes simultaneously while maintaining $\gamma_{1}$ unrenormalized. In this case, a dimensional crossover from $2 \mathrm{D}$ to $\mathrm{OD}$ is induced for $\gamma_{2,3} \rightarrow 0$, leading to two flat energy bands, beyond the merging of the Dirac points $[4,6]$, which occurs at $\left|\gamma_{1}\right|=2\left|\gamma_{2,3}\right|$. A nonzero value of $\gamma^{\prime}$ breaks the particle-hole symmetry and leads to a coupling among the 1D chains and the OD dimers, for the $\gamma_{1}=0$ and $\gamma_{2,3}=0$ cases, respectively, and thus to a weak $2 \mathrm{D}$ dispersion. The merging and the alignment of Dirac points, however, are not affected. Moreover, for a shaking direction parallel to $\mathbf{d}_{1}$, one pair of NNN hopping amplitudes $\left[ \pm\left(\mathbf{d}_{2}-\mathbf{d}_{3}\right)\right]$ remains unrenormalized, and its relative importance is thus enhanced when compared to the decreasing NN hopping amplitudes. In this limit, beyond the semimetallic and the band-insulating phases, an unusual metallic phase can appear that consists of particle and hole pockets with a nonvanishing density of states even at half filling.

Furthermore, we have investigated the role of weak repulsive on-site interactions. The resulting ground state is then adiabatically connected to that of the noninteracting system, and we have self-consistently calculated the dependence of the atomic density on the (local) chemical potential. The density profiles of the different phases, e.g., the gapless semimetal or the gapped band insulator, and the different dimensionalities 
may be determined experimentally by in situ density measurements. Moreover, momentum-resolved Raman spectroscopy might be a promising technique to measure the band structure associated with these different phases.

\section{ACKNOWLEDGMENTS}

We thank Gilles Montambaux, Guangquan Wang, Andreas Hemmerich, and Marco Di Liberto for fruitful discussions. We also thank Christoph Ölschläger for informing us about an error in the calculations. This work was financially supported by the ANR project NANOSIM GRAPHENE under Grant No. ANR-09-NANO-016 and by the Netherlands Organization for Scientific Research (NWO).

\section{APPENDIX: EFFECTIVE HAMILTONIAN}

For the studied case, $H(t)-\hbar \partial_{t} F(t)=H_{0}$, where $H_{0}$ was given in Eq. (2). Since the NN hopping is usually larger than the
NNN hopping, $\gamma^{\prime}<\gamma$, and since we let the chemical potential be in the range $-2 \gamma \leqslant \mu \leqslant 2 \gamma$, the dominant energy scale in the Hamiltonian $H_{0}$ is $\gamma$. Therefore, if $\gamma \ll \hbar \Omega$, the condition (7) is satisfied and the Floquet theory may be applied. [21]

In the general case, the effective Hamiltonian is given by

$$
H_{\mathrm{eff}}=\left\langle\sum_{n=0}^{\infty} \frac{i^{n}}{n !}\left[\hat{F}(t), H_{0}\right]_{n}\right\rangle_{T}
$$

where for the shaken honeycomb lattice, we choose

$$
\hat{F}(t)=\frac{m \Omega^{2}}{\hbar \Omega} \sin (\Omega t)\left(\sum_{\mathbf{r} \in A} \mathbf{r} \cdot \rho a_{\mathbf{r}}^{\dagger} a_{\mathbf{r}}+\sum_{\mathbf{r} \in B} \mathbf{r} \cdot \rho b_{\mathbf{r}}^{\dagger} b_{\mathbf{r}}\right) .
$$

Using the (nonvanishing) commutation relations

$$
\begin{aligned}
{\left[a_{\mathbf{r}^{\prime}}^{\dagger} a_{\mathbf{r}^{\prime}}, a_{\mathbf{r}}^{\dagger} b_{\mathbf{r}+\mathbf{d}_{j}}\right] } & =a_{\mathbf{r}^{\prime}}^{\dagger} b_{\mathbf{r}+\mathbf{d}_{j}} \delta \mathbf{r}^{\prime}, \mathbf{r}, \quad\left[a_{\mathbf{r}^{\prime}}^{\dagger} a_{\mathbf{r}^{\prime}}, b_{\mathbf{r}_{+}+\mathbf{d}_{j}}^{\dagger} a_{\mathbf{r}}\right]=-b_{\mathbf{r}_{+}+\mathbf{d}_{j}}^{\dagger} a_{\mathbf{r}^{\prime}} \delta \mathbf{r}^{\prime}, \mathbf{r}, \\
{\left[a_{\mathbf{r}^{\prime}}^{\dagger} a_{\mathbf{r}^{\prime}}, a_{\mathbf{r}}^{\dagger} a_{\mathbf{r}+\mathbf{d}_{i}-\mathbf{d}_{j}}\right] } & =a_{\mathbf{r}^{\prime}}^{\dagger} a_{\mathbf{r}+\mathbf{d}_{i}-\mathbf{d}_{j}} \delta \mathbf{r}^{\prime}, \mathbf{r}-a_{\mathbf{r}^{\prime}}^{\dagger} a_{\mathbf{r}^{\prime}} \delta_{\mathbf{r}^{\prime}, \mathbf{r}_{+}+\mathbf{d}_{i}-\mathbf{d}_{j},}
\end{aligned}
$$

which are valid for both fermionic and bosonic creation and annihilation operators, and equivalent ones for the creation and annihilation operators on the $B$ sublattice, the multiple commutator in Eq. (A1) becomes

$$
\begin{aligned}
& {\left[\hat{F}(t), H_{0}\right]_{n}=\left[\frac{m \Omega^{2}}{\hbar \Omega} \sin (\Omega t)\right]^{n}\left\{-\gamma \sum_{j=1}^{3} \sum_{\mathbf{r} \in A}\left(\mathbf{d}_{j} \cdot \boldsymbol{\rho}\right)^{n}\left[(-1)^{n} a_{\mathbf{r}}^{\dagger} b_{\mathbf{r}+\mathbf{d}_{j}}+b_{\mathbf{r}_{+} \mathbf{d}_{j}} a_{\mathbf{r}}\right]-\gamma^{\prime} \sum_{i=1}^{3} \sum_{j=1, j \neq i}^{3}\right.}
\end{aligned}
$$

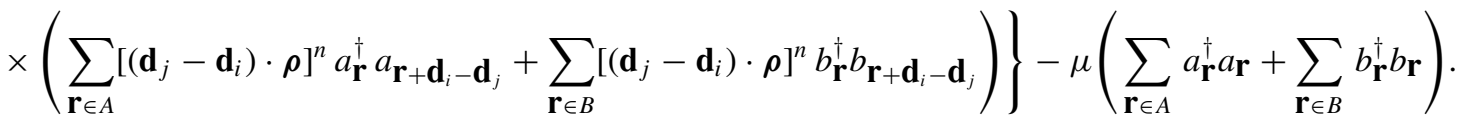

Finally, after performing the time average and evaluating the sum over $n$, the effective Hamiltonian (10) is obtained.

[1] K. S. Novoselov, A. K. Geim, S. V. Morosov, D. Jiang, Y. Zhang, S. V. Dubonos, I. V. Grigorieva, and A. A. Firsov, Science 306, 666 (2004).

[2] C. Berger, Z. Song, T. Li, A. Y. Ogbazghi, R. Feng, Z. Dai, A. N. Marchenkov, E. H. Conrad, P. N. First, and W. A. de Heer, J. Phys. Chem. 108, 19912 (2004).

[3] For recent reviews on graphene, see A. H. Castro Neto, F. Guinea, N. M. R. Peres, K. S. Novoselov, and A. K. Geim, Rev. Mod. Phys. 81, 109 (2009); M. O. Goerbig, ibid. 83, 1193 (2011).

[4] Y. Hasegawa, R. Konno, H. Nakano, and M. Kohmoto, Phys. Rev. B 74, 033413 (2006).

[5] P. Dietl, F. Piechon, and G. Montambaux, Phys. Rev. Lett. 100, 236405 (2008).

[6] G. Montambaux, F. Piéchon, J. N. Fuchs, and M. O. Goerbig, Phys. Rev. B 80, 153412 (2009); Eur. Phys. J. B 72, 509 (2009).

[7] M. O. Goerbig, J. N. Fuchs, G. Montambaux, and F. Piéchon, Phys. Rev. B 78, 045415 (2008); V. M. Pereira, A. H. Castro Neto, and N. M. R. Peres, ibid. 80, 045401 (2009).
[8] C. Becker, P. Soltan-Panahi, J. Kronjäger, S. Dörscher, K. Bongs, and K. Sengstock, New J. Phys. 12, 065025 (2010); A. Klinger, S. Degenkolb, N. Gemelke, K.-A. Brickman Soderberg, and C. Chin, Rev. Sci. Instrum. 81, 013109 (2010); J. Struck, C. Ölschläger, R. Le Targat, P. Soltan-Panahi, A. Eckardt, M. Lewenstein, P. Windpassinger, and K. Sengstock, Science 333, 996 (2011); P. Soltan-Panahi, D.-S. Lühmann, J. Struck, P. Windpassinger, and K. Sengstock, Nat. Phys. 8, 71 (2012).

[9] S. Sorella and E. Tosatti, Europhys. Lett. 19, 699 (1992).

[10] Z. Y. Meng, T. C. Lang, S. Wessel, F. F. Assaad, and A. Muramatsu, Nature (London) 464, 847 (2010).

[11] G. Wang, M. O. Goerbig, Ch. Miniatura, and B. Grémaud, Europhys. Lett. 95, 47013 (2011).

[12] A. Bermudez, N. Goldman, A. Kubasiak, M. Lewenstein, and M. A. Martin-Delgado, New J. Phys. 12, 033041 (2010).

[13] H. Lignier, C. Sias, D. Ciampini, Y. Singh, A. Zenesini, O. Morsch, and E. Arimondo, Phys. Rev. Lett. 99, 220403 (2007). 
[14] A. Zenesini, H. Lignier, D. Ciampini, O. Morsch, and E. Arimondo, Phys. Rev. Lett. 102, 100403 (2009).

[15] M. DiLiberto, O. Tieleman, V. Branchina, and C. M. Smith, Phys. Rev. A 84, 013607 (2011).

[16] K. W. Madison, M. C. Fischer, R. B. Diener, Qian Niu, and M. G. Raizen, Phys. Rev. Lett. 81, 5093 (1998); M. Glück, A. Kolovsky, and H. Korsch, Phys. Rep. 366, 103 (2002).

[17] S. R. White and A. E. Feiguin, Phys. Rev. Lett. 93, 076401 (2004); A. J. Daley, C. Kollath, U. Schollwöck, and G. Vidal, J. Stat. Mech.: Theory Exp. (2004) P04005; D. Gobert, C. Kollath, U. Schollwöck, and G. Schutz, Phys. Rev. E 71, 036102 (2005); U. Schollwöck, Rev. Mod. Phys. 77, 259 (2005); C. Kollath, A. M. Läuchli, and E. Altman, Phys. Rev. Lett. 98, 180601 (2007).

[18] J. K. Freericks, V. M. Turkowski, and V. Zlatić, Phys. Rev. Lett. 97, 266408 (2006); M. Eckstein, M. Kollar, and P. Werner, ibid. 103, 056403 (2009).

[19] M. Rigol, Phys. Rev. Lett. 103, 100403 (2009).

[20] M. Grifoni and P. Hänggi, Phys. Rep. 304, 229 (1998).

[21] A. Hemmerich, Phys. Rev. A 81, 063626 (2010).
[22] A. Eckardt, C. Weiss, and M. Holthaus, Phys. Rev. Lett. 95, 260404 (2005).

[23] A. Eckardt, P. Hauke, P. Soltan-Panahi, C. Becker, K. Sengstock, and M. Lewenstein, Europhys. Lett. 89, 10010 (2010).

[24] K. L. Lee, B. Grémaud, R. Han, B. G. Englert, and Ch. Miniatura, Phys. Rev. A 80, 043411 (2009).

[25] A. Eckardt and M. Holthaus, Phys. Rev. Lett. 101, 245302 (2008).

[26] D. Poletti and C. Kollath, Phys. Rev. A 84, 013615 (2011).

[27] S.-L. Zhu, B. Wang, and L.-M. Duan, Phys. Rev. Lett. 98, 260402 (2007).

[28] P. Soltan-Panahi, J. Struck, P. Hauke, A. Bick, W. Plenkers, G. Meineke, C. Becker, P. Windpassinger, M. Lewenstein, and K. Sengstock, Nat. Phys. 7, 434 (2011).

[29] T. L. Dao, A. Georges, J. Dalibard, C. Salomon, and I. Carusotto, Phys. Rev. Lett. 98, 240402 (2007); T. L. Dao, I. Carusotto, and A. Georges, Phys. Rev. A 80, 023627 (2009).

[30] J. Stewart, J. Gaebler, and D. Jin, Nature (London) 454, 744 (2008).

[31] P. T. Ernst, S. Götze, J. S. Krauser, K. Pyka, D.-S. Lühmann, D. Pfannkuche, and K. Sengstock, Nat. Phys. 6, 56 (2010). 